\title{
ИССЛЕДОВАНИЕ МЕХАНИЗМОВ ВЗАИМОДЕЙСТВИЯ СУБЪЕКТОВ ЦИФРОВЫХ ЭКОНОМИЧЕСКИХ СИСТЕМ
}

\author{
() 2020 Родионов Дмитрий Григорьевич \\ доктор экономических наук, профессор \\ Высшая инженерно-экономическая школа \\ Санкт-Петербургский политехнический университет Петра Великого (СПбПУ), \\ Россия, Санкт-Петербург \\ E-mail: dmitry.rodionov@spbstu.ru \\ (C) 2020 Конников Евгений Александрович \\ кандидат экономических наук, Высшая инженерно-экономическая школа \\ Санкт-Петербургский политехнический университет Петра Великого (СПбПУ), \\ Россия, Санкт-Петербург \\ E-mail: konnikov.evgeniy@gmail.com \\ (c) 2020 Сергеев Дмитрий Анатольевич \\ кандидат экономических наук, доцент \\ Высшая инженерно-экономическая школа \\ Санкт-Петербургский политехнический университет Петра Великого (СПбПУ), \\ Россия, Санкт-Петербург \\ E-mail: sergeev_da@spbstu.ru
}

Гиперконкуренция на мировых В2С рынках, неуклонное повышение доступности и скорости технологий обмена информацией, а также трансформация паттернов взаимодействия потребителей мультипликативно воздействуют на процесс цифровизации экономических систем. На микроуровне данный процесс проявляется в цифровизации отдельных бизнес-процессов, в то время как на макроуровне данный процесс проявляется в масштабной трансформации взаимодействия социально-экономических систем. Следствием данных процессов стало формирование цифровой надстройки современной экономики, сущностно являющейся системой как обмена, так и генерации экономической информации, описывающей вещественную реальность. При этом, состав субъектов взаимодействия в данной среде единообразен, однако механизмы их взаимодействия трансформируются в следствии как структуры генерируемой и потребляемой информации, так и скорости и широты ее распространения. В рамках данного исследования авторы предлагают систему дифференциальных уравнений, описывающих взаимодействие ключевых субъектов цифровой экономической системы, представляющую собой модель модифицированного хаотического аттрактора Чуа.

Ключевые слова: цифровая экономическая система, генераторы блага, удовлетворённость потребителей, аттрактор.

Процесс цифровизации экономики на данный момент является объективной реальностью, значительно медиирующийся ограничениями, налагаемыми условиями существования в рамках пандемии COVID-19. Данный процесс существует как на микроуровне, находя свое отражение в цифровизации отдельных бизнеспроцессов, так и на макроуровне, проявляюсь в трансформации механизмов взаимодействия социально-экономических систем. Процесс цифровизации способствует формированию параллельно существующей экономической среды - цифровой экономической среды. Данная среда формируется подобно экономической надстройке, и представляет из себя совокупность информационных массивов и механизмов, позволяющих реализовывать экономическое взаимодействия полностью или частично вне объективного мира. При этом, по мере развития данной надстройки, она начинает оказывать воздействие на образующий ее базис - вещественную экономическую системы, или объективную реальность. Математическое описание законов взаимодействия субъектов цифровых 
экономических систем имеет крайне дискуссионный характер. В первую очередь, это обусловлено многомерностью и иерархической неоднозначностью данных систем. В условиях рыночного взаимодействия, можно выделить 3 основные группы участников, дифференцированные в соответствии с характером их интересов и выгод:

1. Генераторы конечного блага и его составных элементов. Данные группа представлена предприятиями и организациями, производящими продукты и оказывающие услуги иным участникам цифровых экономических систем, а также производящими продукты и оказывающие услуги, необходимые для формирования конечного блага. Количественное выражение интересов представителей данной группы может дифференцироваться, однако наиболее универсальной количественной характеристикой может выступать прибыль.

2. Потребители конечного блага. Данная группа представлена потребителями - клиентами генераторов конечного блага. Надо отметить, что представители данной группы в рамках цифровых экономических систем могут сочетать в себе также роль генераторов конечного блага. Количественное выражение интересов представителей данной группы может быть выражено относительным коэффициентом удовлетворенности. Коэффициентов удовлетворенности потребителей в маркетинге существуют крайне много. В данном случае для целей универсализации данный показатель можно представить в виде ранговой переменной, отражающей степень соответствия ожиданий потребителя от потребления того или иного продукта или услуги её фактическим характеристикам.

3. Администратор цифровой экономической системы. Несмотря на то, что цифровая экономическая система может формироваться за счет взаимодействия описанных ранее групп участников, она формируется в нормативноправовом поле, что определяет наличие законов и правил взаимодействия. Более того, инфраструктура цифровой экономической системы может формироваться принудительно, а архитектором и последующим администратором может выступать государство или крупные корпоративные участники экономической системы, для которых сама инфраструктура и процесс ее контроля выступают в качестве генерируемого блага. Данная группа не имеет четко выражен- ного вектора интересов, вследствие чего целесообразно отразить влияние вариативных параметров воздействия представителей данной группы участников цифровой экономической системы на интересы иных групп участников. Количественно выразить данной влияние может индикатор уровня контроля, отражающий степень контролируемости цифровой экономической системы со стороны ее администраторов.

Таким образом, в качестве количественных показателей, взаимодействие которых отражает математические законы функционирования цифровой экономической системы можно выделить:

- Прибыль предприятий - генераторов конечного блага (X).

- Коэффициент удовлетворенности потребителей конечного блага (Y).

- Индикатор уровня контроля системы со стороны администратора (Z).

Для целей моделирования предположим, что данные показатели стандартизированы, и измеряются в относительных единицах измерения. В первую очередь, надо отметить диалектичный характер взаимодействия администратора цифровой экономической системы и потребителей конечного блага. Приращение параметра контроля неизменно снижает уровень адаптивности цифровой экономической системы, снижает скорость принятия решений, а также повышает расход ресурсов, связанных с обеспечение деятельности администратора. Данные процессы приводят к снижению ассортимента, снижению адаптивности взаимодействия и также повышению стоимости конечного блага. Таким образом, изменение уровня контроля системы со стороны администратора может быть определено обратно коэффициенту удовлетворенности потребителей конечного блага, и выражено следующим дифференциальным уравнением:

$$
\frac{d z_{t}}{d t}=-\beta * y_{t}
$$

Где:

1. $Z_{t}-$ стандартизированный индикатор уровня контроля системы со стороны администратора в периоде $\mathrm{t}$;

2. $Y_{t}-$ стандартизированный коэффициент удовлетворенности потребителей конечного блага в периоде $\mathrm{t}$;

3. $\beta$ - коэффициент эластичности административного контроля по удовлетворенности 
потребителей конечного блага. Данный коэффициент отражает степень чувствительности системы администрирования по отношению к удовлетворенности потребителей.

Взаимодействие генераторов и потребителей конечного блага носит динамическинелинейных характер. С одной стороны, повышение уровня удовлетворенности потребителей медиирует прирост прибыли в относительно последующем периоде, за счет приращения спроса, обеспеченного распространением информации относительно колебаний удовлетворенности. Однако, так как уровень удовлетворенности базируется на ожиданиях потребителей, в последующем периоде данный показатель будет завышен, что в свою очередь может снизить уровень удовлетворенности. Выразить данное взаимодействие можно посредствам интеграции тригонометрической функций в описываемое дифференциальное уравнение:

$$
\frac{d x_{t}}{d t}=\alpha *\left(y_{t}+b * \sin \left(\frac{\pi x_{t}}{2 a}\right)\right)
$$

Где:

1. $X_{t}-$ стандартизированная прибыль генераторов конечного блага в периоде $t$;

2. $\alpha-$ коэффициент эластичности прибыли генераторов конечного блага по удовлетворённости потребителей. Данный коэффициент отражает степень чувствительности рынка по отношению к удовлетворенности потребителей;

3. $b-$ коэффициент эластичности удовлетворенности потребителей к ретроспективным колебаниям удовлетворенности. Данный показатель отражает степень трансформации ожиданий потребителей под воздействием текущей их удовлетворенности;

4. $\quad a-$ индикатор скорости трансформации потребительских ожиданий. Данный индикатор отражает частоту трансформации потребительских ожиданий за период времени. Сравнительно малые значения данного индикатора отражают низкий уровень лояльности потребителей, в то время, когда высокие значения данного показателя выражают относительную, зрелось потребителей, и их готовность развивать цифровую экосистему совместно с генераторами конечного блага.

Изменение удовлетворенности потребителей конечного блага в свою очередь может быть линейно зависима от всех трех выделенных по- казателей. Приращение стандартизированной прибыли прямо пропорционально изменению стандартизированного коэффициента удовлетворенности потребителей конечного блага. При этом приращение стандартизированного индикатора уровня контроля системы со стороны администратора также способствует повышению удовлетворенности, так как уровень ожиданий потребителей снижается, что значительно повышает конверсию изменений. Таким образом, данную зависимость можно выразить посредствам следующего дифференциального уравнения:

$$
\frac{d y_{t}}{d t}=x_{t}-y_{t}+z_{t}
$$

Сформированная система дифференциальных уравнений позволяет сформировать множество неустойчивых траекторий, обладающих хаотическим характером. Малые возмущения данной системы могут экспоненциально трансформироваться. Таким образом, данной системе свойственен динамический хаос, и образует странный аттрактор. Архитектура сформированного аттрактора идентична архитектуре модифицированного хаотического аттрактора Чуа [1]. Решение приведенной системы уравнений при различных комбинациях значений коэффициентов образует множество неустойчивых траекторий. Приведем пример для линейного изменения значений $\mathrm{t}$ от 0 до 100, образующего 40000 наблюдений. Базовые значения показателей $\mathrm{x}_{\mathrm{t}}, \mathrm{y}_{\mathrm{t}}$, и $\mathrm{z}_{\mathrm{t}}$ составляют 1,1 и 0 соответственно. Базовые значения коэффициентов $\beta, \alpha, b$ и $a$ составляют $14.286,10.82,0.11$ и 1.3 соответственно. На рисунке 1 представлено базовое состояние прилагаемого аттрактора.

В соответствии с векторами трансформации можно однозначно говорить о следующих законах функционирования описываемой системы взаимодействия:

1. Прибыть генераторов конечного блага находится в обратной зависимости с индикатором уровня контроля системы. Следовательно, для целей приращения прибыли в рамках цифровой экономической системы необходима ее либерализация, выраженная в планомерном снижении уровня контроля со стороны администратора.

2. Уровню удовлетворённости потребителей конечного блага свойственны гармонические колебания, что определяет возможность по- 
28

Экономические науки • 2020 • № 10 (191)

Начальные условия: $[1,1,0]$

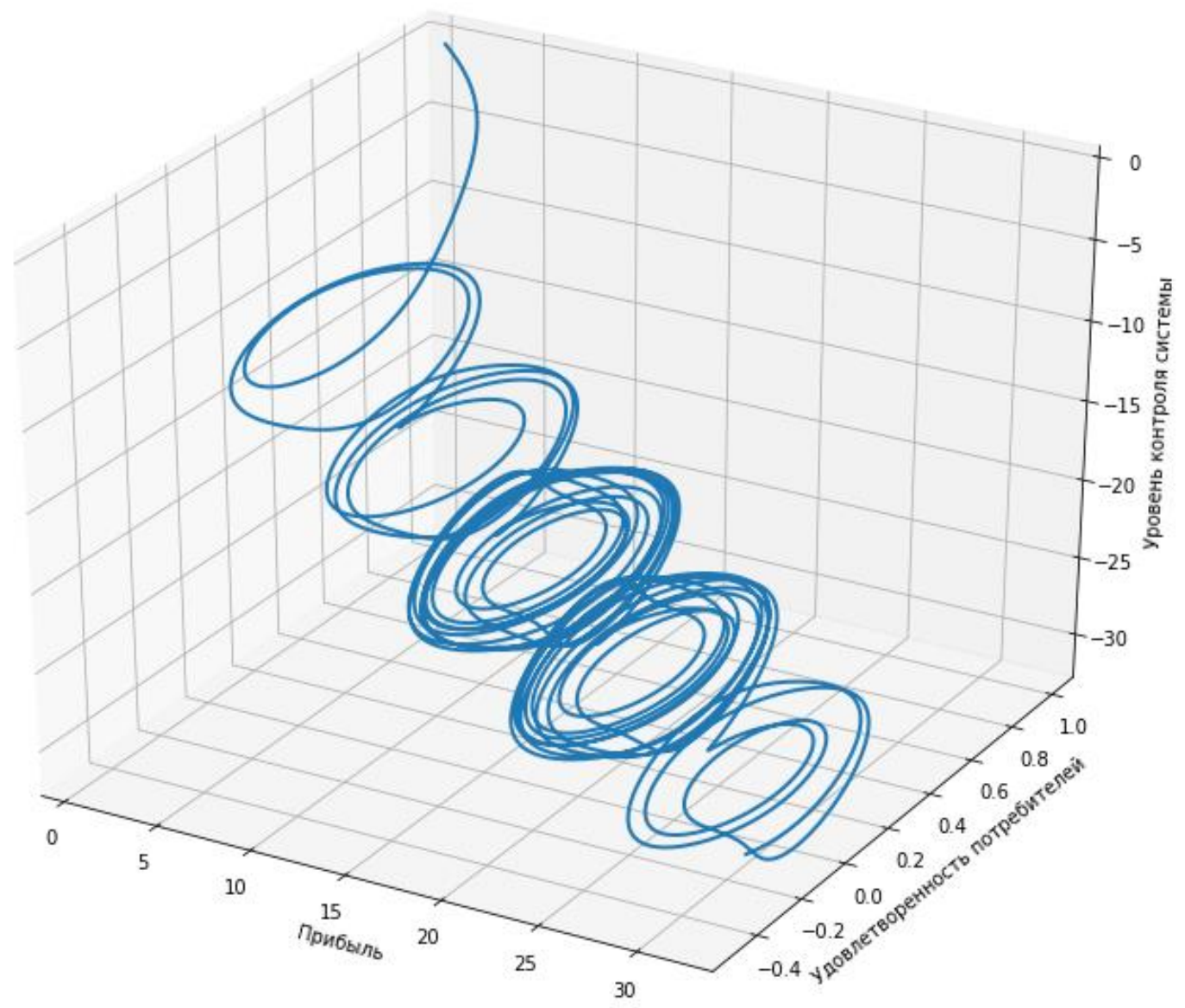

Рисунок 1. Базовое состояние аттрактора

лучения различного уровня удовлетворенности при идентичных значениях прибыли. В первую очередь это обусловлено динамикой ожиданий потребителей, которые в условиях цифровой экономической системы универсализируются за счет насыщенности информационной среды и доступности коммуникационных каналов.

Для целей подтверждения выдвинутых законов следует смоделировать изменения показателей эластичности. На рисунке 2 представлено трансформация состояния аттрактора при изменении эластичности административного контроля по удовлетворенности потребителей конечного блага.

Как можно видеть, по мере увеличения эластичности административного контроля по удовлетворенности потребителей конечного блага усложняется структура колебаний удовлетворённости потребителей по изменению прибыли. При этом, описанные ранее общие законы трансформации остаются неизменными. Следо- вательно, по мере увеличения чувствительности системы администрирования к колебаниям удовлетворенности потребителей, значительно возрастает дельта удовлетворённости, приобретая форму тригонометрической функции. Таким образом, можно утверждать и обратную тенденцию, в соответствии с которой, снижение чувствительности системы администрирования к колебаниям удовлетворенности потребителей, стабилизирует уровень удовлетворённости потребителей. Далее рассмотрим трансформацию состояния аттрактора при изменении эластичности прибыли генераторов конечного блага по удовлетворённости потребителей (рисунок 3).

Как можно видеть на рисунке, увеличение чувствительности прибыли генераторов конечного блага к изменению удовлетворённости потребителей в значительной мере центрирует данный показатель. Следовательно, по мере повышения значимости уровня удовлетворенности потребителей для генераторов блага, уве- 

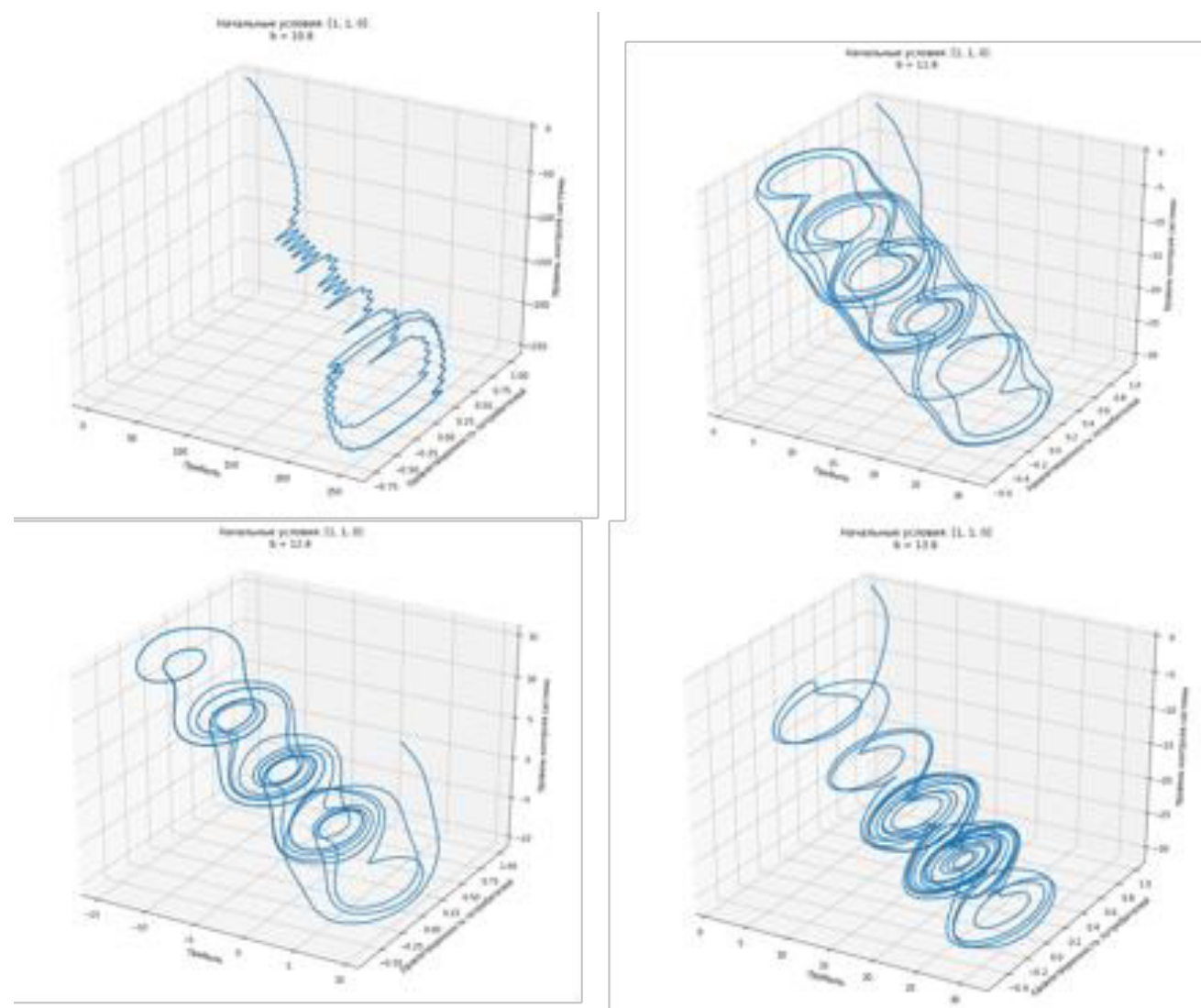

Рисунок 2. Трансформация состояния аттрактора при изменении эластичности административного контроля по удовлетворенности потребителей конечного блага (10.8-13.8)

Начальные условия: $[1,1,0]$
$\mathrm{a}=1$

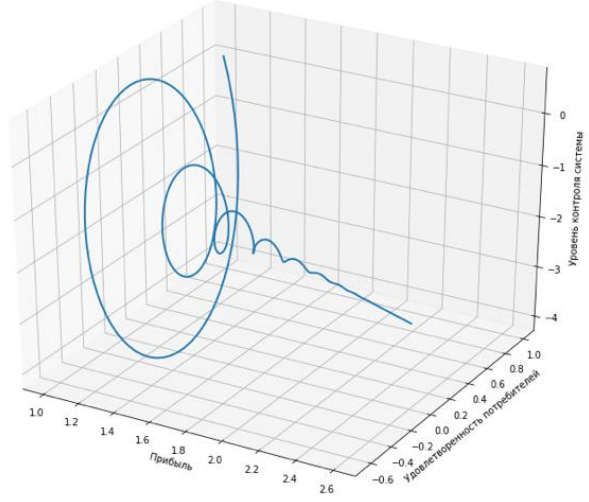

Начальные условия: $[1,1,0]$
$\underset{a}{a}=6$

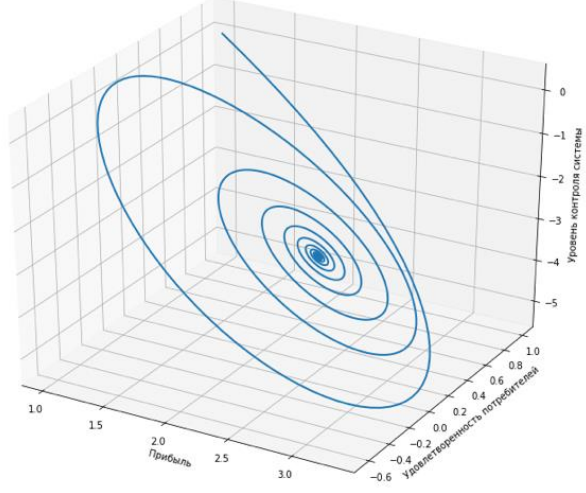

Начальные условия: $[1,1,0]$
$\mathrm{a}=4$

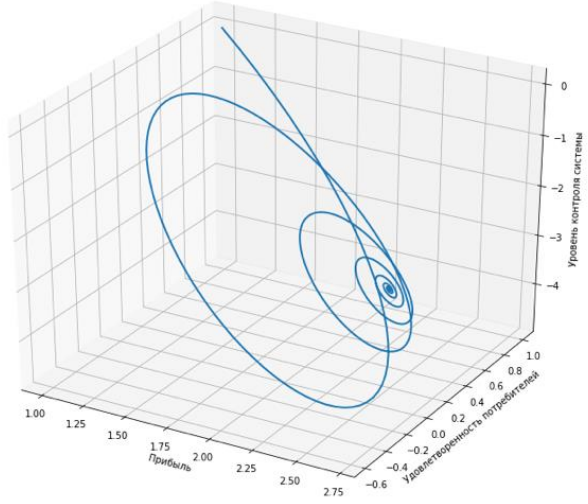

Начальные условия:
$\underset{a}{\mathrm{a}}=8$

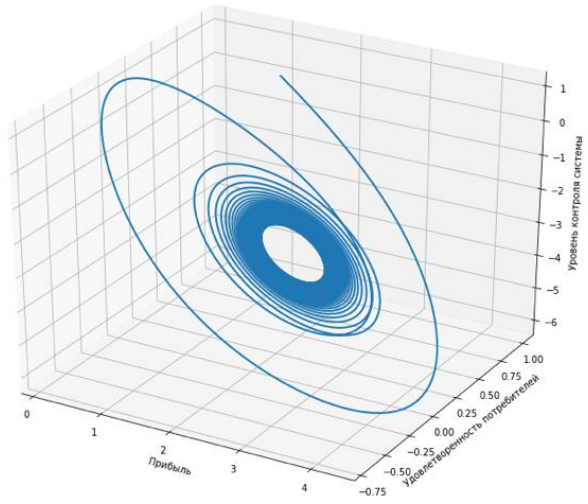

Рисунок 3. Трансформация состояния аттрактора при изменении эластичности прибыли генераторов конечного блага по удовлетворённости потребителей (1-8) 
личивается вариативность удовлетворенности потребителей. Данный механизм во многом объясняется реакцией самих потребителей на изменение потребительских свойств блага. Потребитель адаптируется к скорости реакции гираторов блага и как следствие совершенствует механизмы трансформации своих потребительских ожиданий, следствием чего является увеличение вариативности данного параметра. При этом, выдвинутые ранее базовые тезисы не нарушаются. В завершении рассмотрим изменение состояния аттрактора при изменении эластичности удовлетворенности потребителей к ретроспективным колебаниям удовлетворенности (рисунок 4).

Как можно видеть, при изменении данного показателя, скорость реакции потребителей значительно замедляется, что в свою очередь приводит к относительному увеличению зави- симости прибыли генераторов конечного блага от удалённости потребителей. Данное свойство может быть описано посредствам экстраполяции свойства «деловой репутации». При этом, выдвинутые ранее базовые тезисы не нарушаются.

Таким образом, посредствам сформированной системы дифференциальных уравнений были математически формализованы экономические законы цифровой экономической системы. Основываясь на данных законах, можно утверждать, что первичную значимость для целей развития приобретает цифровой экономической системы приобретает уровень либерализации взаимоотношений субъектов системы и способность генераторов управлять лояльностью потребителей как в тактической, так и в стратегической перспективе.
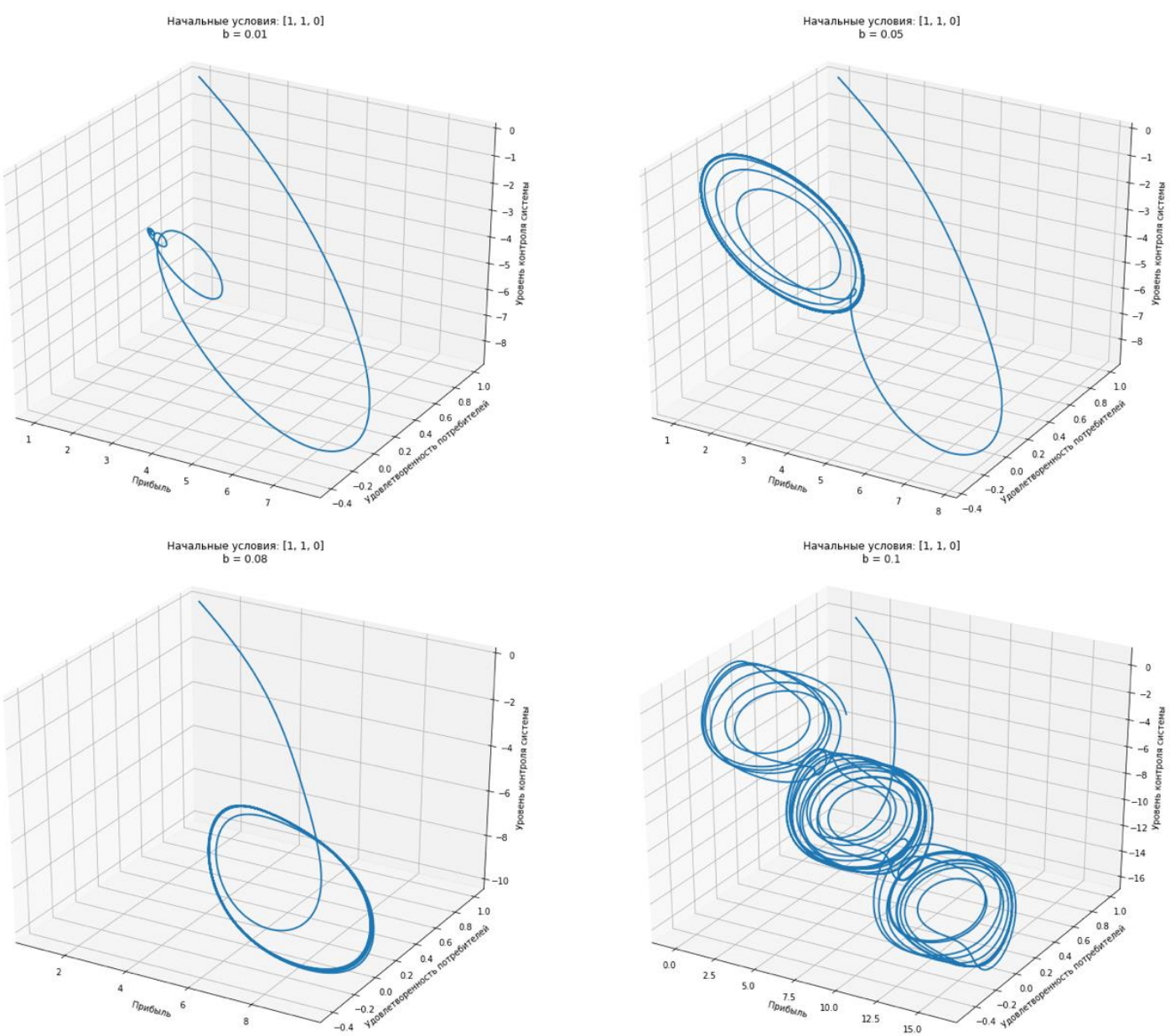

Рисунок 4. Трансформация состояния аттрактора при изменении эластичности удовлетворенности потребителей к ретроспективным колебаниям удовлетворенности (0.01-0.1) 


\section{Библиографический список}

1. Chen Guanrong, Jinhu $\mathrm{Lu}$ «Generating multiscroll chaotic attractors: theories, methods and applications» // International Journal of Bifurcation and Chaos. 16 (4). 2006. С. 793-794.

2. Багоян Е.Г., Демьянец М.В., Десятниченко Д. Ю. и др. Основы цифровой экономики // учебник для и практикум для вузов / Москва, 2020. Сер. Высшее образование

3. Конникова О.А., Конников Е.А. Синтаксический анализ поведения потребителей в цифровой среде // В книге: Маркетинг сотворчества и глобальные коммуникации доверия. Багиев Г. Л., Юлдашева О. У., Шубаева В. Г. и др. Посвящается 90-летию СПбГЭУ и 30-летию кафедры маркетинга. Санкт-Петербург, 2020. С. $132-145$.

4. Родионов Д.Г., Ялымов С. В., Конников Е.А. Влияние информационной среды на субъекты малого и среднего предпринимательства // Экономические науки. 2020. № 189. С. 86-91.

5. Фриц В., Натараян Р., Аренков И. А. и др. Инновации маркетинговых моделей российских компаний в эпоху диджитал трансформации // Коллективная монография / Санкт-Петербург, 2018. 